\title{
Coexistence of a septic state of unspecified etiology with an acute psychotic episode under treatment. Case Study
}

\author{
Anatolie Ţîmbalari, Ilinca Untu, \\ Alexandra Boloṣ, Roxana Chiriță
}

\begin{abstract}
Anatolie Țîmbalari - M.D., resident in psychiatry, "Socola” Institute of Psychiatry, Iaşi, Romania Ilinca Untu - M.D., Ph.D. Student, “Grigore T. Popa” University of Medicine and Pharmacy Iaşi, "Socola" Institute of Psychiatry, Iaşi, Romania

Alexandra Boloș - M.D., Ph.D., lecturer, "Grigore T. Popa” University of Medicine and Pharmacy, Iași

Roxana Chiriță - Professor, M.D., Ph.D., "Grigore T. Popa" University of Medicine and Pharmacy, Iași, "Socola” Institute of Psychiatry, Iaşi, Romania
\end{abstract}

\begin{abstract}
Acute psychotic disorders are psychiatric disorders with various clinical manifestations and variable onset form, which require the administration of psychotropic medication, the first therapeutic line being antipsychotics, which can lead to a number of side effects, some of which are extremely rare, but with systemic resonance. Such a mental illness does not bring with it the guarantee of protection against other general medical conditions, although the collective mind often tends to attribute any manifestation to psychosis or its specific treatment. Thus, therapeutic approaches may be delayed or they may be on the wrong track. This paper aims to present a complex case, challenging in terms of positive diagnosis and differential diagnosis, which intertwine the actual manifestations of an acute psychotic disorder with symptoms of schizophrenia and the multisystemic symptoms of sepsis with unknown etiology, in the context of the administration of psychotropic treatment, which raised the initial suspicion of the appearance of a neuroleptic malignant syndrome. The present case is an eloquent example of the overlap of a general medical condition in a person suffering from an acute episode with multiple psychiatric symptoms, for which has been initiated specialized treatment, thus raising multiple problems of discernment between the elements strictly related to pathology and medication and those associated with organic pathology, which appeared independently of the first. In addition, it challenges us to ask questions about cases of psychosis that can be easily overlooked by people with a certain
\end{abstract}





behavioral pattern and about distrust in psychiatric and medical services in general, in the context of the coincidence of a major medical condition., such as sepsis, which brings with it the tolerance of symptoms both by the person concerned and by those close to him, with a dramatically low or even absent therapeutic adherence.

\section{KEY WORDS:}

Acute psychotic disorder, sepsis, neuroleptic malignant syndrome.

\section{THEORETICAL BACKGROUND}

Infectious conditions and mental disorders are two of the most representative public health problems globally (1). Although a possible correlation between mental disorders and infectious diseases has long been foreshadowed, the alleged genetic link between the two has not been certified by extensive studies (2, 3). Interindividual differences influence the susceptibility to the development of septic status, which may depend on environmental and social factors, vulnerable periods based on psychological stress or low immunity and the genetic profile of the individual (4). Sepsis is often associated with or preceded by other general medical conditions $(1,5)$. Thus, medical conditions that associate common symptoms with those of sepsis should be considered and excluded. Early diagnosis and implicit, rapid initiation of treatment, is a key element in reducing mortality and morbidity associated with sepsis $(3,4)$.

Acute psychotic disorders are psychiatric disorders that can occur as isolated episodes or, most commonly, can crystallize into any other major psychiatric condition, such as schizophrenia and spectrum disorders and affective disorders with psychotic symptoms. In order to establish such a diagnosis, it is necessary to exclude any organicity that could explain the symptoms, as well as the exogenous toxic origin of the appearance of the signs (consumption of psychoactive substances). The first therapeutic line for acute psychotic disorders is antipsychotic medication. Even second-generation antipsychotics can cause a number of side effects, the most important and most severe systemic impact on the patient is the neuroleptic malignant syndrome associated with high fever, hypertension, marked increase in total $\mathrm{CK}$, neurological symptoms, etc.

\section{CASE PRESENTATION}

The present case concerns a 22-year-old male patient, on his first admission to the psychiatric emergency department, without any other documented somatic or psychiatric history. Admission was made in the context of the insidious installation of a symptomatology characterized by hypobulia, mixed sleep disorders, loss of appetite, marked suspicion, bizarre behavior, apathy, delusional ideation of influence, complex auditory hallucinations and pseudohalucinations, the content of which provides additional details, severe integrativerelational deficit, which culminated in the emergence of suicidal ideation with frankly delusional motivation, not accompanied by negative hyperthimia, but by a reduced emotional reactivity.

The objective physical examination, performed at hospitalization, does not reveal pathological elements, the patient being afebrile, hemodynamically stable and not bringing somatic accusations.

Because the patient was hospitalized on a Friday, outside the hours of the analysis 
(2)

laboratory of the medical institution (which is not active during the weekend), the first set of tests was performed three days after admission to the hospital, CBC, ionogram, renal and hepatic function, being in normal parameters. The toxicological examination does not reveal the use of psychoactive substances, and the patient denies the use of drugs and alcohol.

The anamnesis was performed both with the help of the patient and especially with that of his mother, who provided significant details on the premorbid background, intra-family relationships and socio-educational functioning of the patient. Thus, there are declining school performances, starting with the age of 16, the patient being still, at the time of admission, a student in the last year of high school, but in the evening curriculum, sterile concern for abstract fields, obsessive ideas about futility of human existence, suspicion, bizarre behavior, social withdrawal, self-isolation, introversion, minimal and faulty communication, both with his grandparents and his mother. At the same time, we find out that he does not know his father, being the product of an extramarital affair. However, he comes from a family of intellectuals with above average financial possibilities. According to his mother, the patient's natural father, was diagnosed at the age of 40 with persistent delusional disorder, and his paternal grandfather's brother with paranoid schizophrenia. The whole procession of psychiatric symptoms crystallized in a real behavioral pattern, was explained by the family, due to disturbed attachments and vicissitudes of his living environment and conducted to not suspecting a psychiatric pathology and not addressing him to a specialized service. After the death of his grandfather and later, of his grandmother, the exposed behavioral features were accentuated, but the mother continued to consider him a special and sensitive child. Only after the installation of suicidal ideation and in the context of the bizarre nature of its motivation (starting from a delusional core of influence that convicts the patient that a superior force requires him to sacrifice for the good of humanity - the patient says he did not act only for the fact that the life and existence of all people is in vain), the patient was addressed by his mother to the psychiatric emergency service.

The psychological examination performed five days after hospitalization showed persistent autolytic ideation, atypical/discordant manifestations, against the background of an immature, introverted psychostructure, with weak mechanisms of self-regulation and instinctual self-control, social avoidance / isolation and major integrative-relational deficit in the sociofamily environment. The P.A. test, which reflects the accentuated personality traits, revealed: $\quad 75 \%$ anxiety, $50 \%$ demonstrativeness, $50 \%$ hyperexactness, $50 \%$ impulsivity.

The patient performed an electroencephalogram, which showed a normal path, electrocardiogram and internal medicine examination, being in normal parameters.

Thus, corroborating the clinical-anamnestic data and the paraclinical examinations, the diagnosis of Acute Psychotic Disorder with Symptoms of Schizophrenia is established (anamnestic data being relevant for a future diagnosis of Schizophrenia).

He received initiation treatment with atypical antipsychotics (Olanzapine 5mg) and benzodiazepines to obtain the anxiolytic effect (Lorazepam 1mg x 3/day). The dose of the antipsychotic was progressively increased 


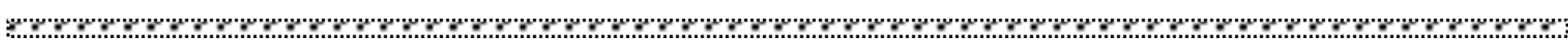

to $15 \mathrm{mg}$ over 7 days without the appearance of extrapyramidal effects, sedation or hypotension, with complete regulation of sleep disorders and a discrete positive effect on communication and correction of perception disturbances.

Seven days after admission, the patient complains of muscle pain, predominantly in the right forearm, no local inflammatory signs, discrete photophobia (subjectively reported by the patient and observed from his pantomime), fever (temperature-39.2 degrees Celsius), discrete increase of CK, but without objective signs of impregnation, which could have occurred in the context of antipsychotic treatment. The doctor on duty at the Infectious Diseases Hospital was contacted, the case was presented and an opinion was received for a specialist consultation. The patient was also referred for craniocerebral computed tomography evaluation and neurological examination. After performing the specialist consultations, it was found that the imaging examination excluded the acute brain lesions, without highlighting any other particular structural changes, the neurological evaluation excluded the focus or meningeal signs, assuming that the febrile syndrome was caused by the administration of antipsychotics, the consultation performed by the infectious disease specialist only raised the suspicion of a virus. Pulmonary stetacoustic, the patient did not present semiological pathological elements, and the chest radiograph did not reveal any change. The brief urine test is within normal parameters. In this context, the antipsychotic is discontinued, benzodiazepines are maintained and perfusions and antipyretic medication are administered, with an apparent favorable evolution of the somatic condition.

Ten days after admission, the patient complains of pain in the right olecranon level, the related region showing inflammatory signs. He reports a possible trauma at this level prior to hospitalization. The radiography performed does not detect cubito-humeral radiological changes. Balneological consultation is also performed, recommending anti-inflammatory treatment with ibuprofen. The febrile state remained present, so that the administration of anti-inflammatory and antipyretic medication was continued, without obtaining a remarkable decrease in temperature (but values between 37.5-38.0 degrees Celsius).

Twelve days after admission, the patient presents with suddenly installed jaundice, myalgia, inflammation in all joints of the limbs (both upper and lower), with partial associated functional impotence, maintaining a subfebrile condition under antipyretics. Laboratory analyzes collected and monitored in dynamics, reflect a dramatic increase in transaminases, total bilirubin with double the upper limit of normal and discrete nitrogen retention syndrome, leukocytosis with neutrophilia and urine culture that does not show a pathogen that suggests a point of urinary output. It should be mentioned that in the previous days, all these values were within normal limits. The patient had no wounds or other skin lesions with potential for infection.

At the same time, it was present at cardiac auscultation, tachycardia (heart rate 140 beats/minute, peripheral pulse synchronous with the central one), chest pain, described by the patient as located retrosternally, with lowmedium intensity and low blood pressure up to $80 / 50 \mathrm{mmHg}$. The patient was sent to the main emergency hospital in the county, with the presumption of diagnosis, Sepsis of unspecified etiology, being detained in the Department of Anesthesia and Intensive Care for additional examinations and specialized 


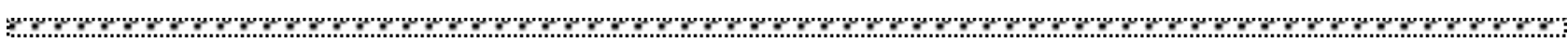

treatment. The main suspicion of admission to

this clinic was malignant neuroleptic syndrome, pending the results of blood culture. However, the patient's general symptoms did not correspond to this diagnosis, except within the presence of resistant febrile syndrome and mild hypertonia with progressive onset. After a series of investigations carried out in order to establish a definite diagnosis, however, staphylococcal sepsis was objectified, the entry path not being able to be highlighted. After broad-spectrum antibiotic therapy and supportive treatment, the patient's evolution was a slow one, being subsequently timed for another week in an internal medicine department, for complete somatic evaluation.
He was finally discharged, fully recovered, with the recommendation to return to the psychiatric service to continue psychotropic treatment, consistent with the diagnosis of Acute Psychotic Disorder. However, both the patient and his mother refused to be referred to the Institute of Psychiatry, returning home. The patient also rejected the possibility of outpatient psychiatric dispensary. His mother kept in touch with his doctor for a period of time, reporting that the symptoms prior to the initial hospitalization persist, but that the patient no longer describes suicidal ideation. From their statements, the symptoms seemed absolutely manageable at home, the patient behaving in the same way as before the symptoms worsened and he went to the psychiatric service.

\section{DISCUSSIONS AND CONCLUSIONS}

The present case once again raises the issue of the involvement of mental disorders in the precipitation of infectious states, through the associated mental stress. The patient in question had for at least six years a behavioral pattern suggestive of the insidious onset of a major mental disorder. At the time of hospitalization and another seven days later, no other medical condition that could explain the appearance of psychiatric symptoms has been objectified, the onset of the clinical picture that was certified as sepsis occurring one week after hospitalization. At the same time, the patient's family environment and interpersonal relationships, limited only to his family members, as well as the disturbed attachment and the atypical family of origin, must be brought back into discussion.

On the other hand, it is interesting to note that the first suspicion of specialists belonging to other medical specialties was the neuroleptic malignant syndrome, which was finally refuted, in the context in which the entrance gate of the infectious agent could not be highlighted.

It is important to emphasize that any psychiatric pathology, including acute psychotic disorders do not exempt the patient from exposure to other general medical conditions, and the new generation psychotropic medication has a good safety profile, and can give severe side effects relatively rarely.

The whole context to which the patient was exposed, strengthened his belief, as well as that of his mother, that he does not need specialized help and that he can return to his pseudo-normality and daily routine.

The present case has multiple facets that make us meditate on the level of knowledge of the general population on the discernment of the normal from the abnormal and the willingness to seek specialized help, differential diagnosis problems and delayed therapeutic intervention in severe 

somatic diseases, in the context in which it is tried to exclude some iatrogens with origin in the administration of psychiatric medication $(6,7)$.

\section{ACKNOWLEDGEMENTS AND DISCLOSURES}

The authors declare that they have no potential conflicts of interest to disclose.

\section{REFERENCES}

1. Rohleder N.(2014). Stimulation of systemic low-grade inflammation by psychosocial stress. Psychosomatic medicine.; 76:181-189. [PubMed: 24608036]

2. Cohen S, Kamarck T, Mermelstein R. (1983). A global measure of perceived stress. Journal of health and social behavior.; 24:385-396. [PubMed: 6668417]

3. Viscoli C. (2016).Bloodstream infections: the peak of the iceberg. Virulence.;7:248-51

4. Contou D, Roux D, Jochmans S, et al. (2016) Septic shock with no diagnosis at 24 hours: a pragmatic multicenter prospective cohort study. Crit Care.;20:360.

5. Burg MM, Edmondson D, Shimbo D, Shaffer J, Kronish IM, Whang W, Alcantara C, Schwartz JE, Muntner P, Davidson KW.(2013). The 'perfect storm' and acute coronary syndrome onset: do psychosocial factors play a role? Progress in cardiovascular diseases.; 55:601-610.[PubMed: 23621970]

6. Glaser R, Rabin B, Chesney M, Cohen S, Natelson B. (1999).Stress-induced immunomodulation: implications for infectious diseases? JAMA.; 281:2268-2270. [PubMed: 10386538]

7. Hernandez C, Cobos-Trigueros N, Feher C, et al.(2014) Community-onset bac- teraemia of unknown origin: clinical characteristics, epidemiology and outcome. Eur J Clin Microbiol Infect Dis.;33:1973-80.

\section{Correspondence:}

Alexandra Boloş,

M.D., Ph.D., lecturer, “Grigore T. Popa” University of Medicine and Pharmacy,16 Universităţii street, Iași

Submission: 25 sep 2020

Acceptance: 29 nov 2020 